\title{
Spontaneous regression of multiple pulmonary aneurysms in a patient with midaortic syndrome and arterial hypertension
}

\author{
Kardos M, Masura J
}

Department of Functional Diagnostics, Children’s Cardiac Center, Bratislava, Slovakia. kardi.marek@gmail.com

To the Editor,

Pulmonary arterial pseudoaneurysms (PAPs, Rasmussen's aneurysms) are rare, but sometimes fatal complications of pulmonary diseases, have classicaly been asociated with the cavitary lesions of reactivation tuberculosis. But they can also occur as complication of endocarditis, pulmonary abscess or catheter infections. A pseudoaneurysm does not involve all layers of the arterial wall and is therefore at higher risk of rupture. In massive hemoptysis surgical treatment or coil/ethylene vinyl copolymer embolisation are methods of choice (1). Assessment with contrast-enhanced CT allows accurate evaluation of pulmonary artery pseudoaneurysms,

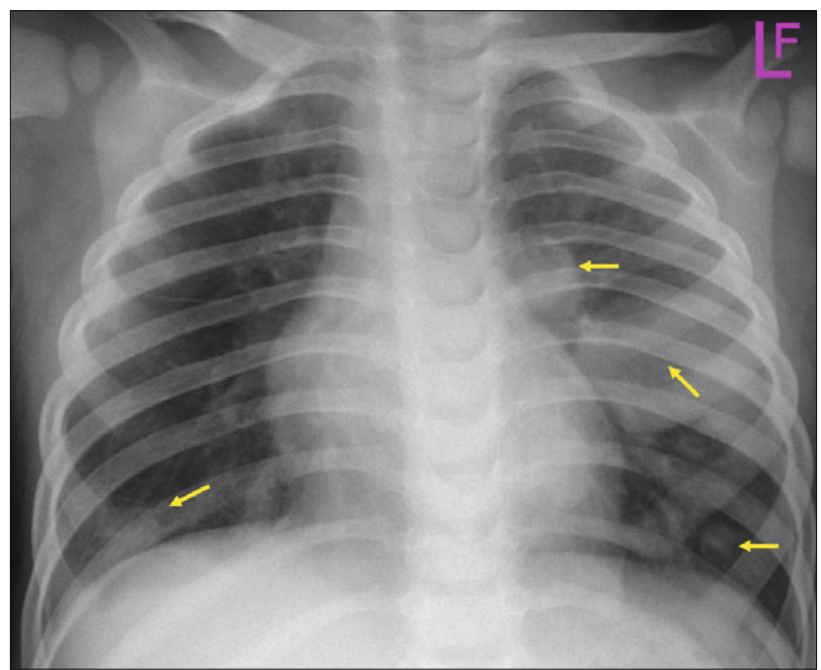

Fig. 1. X-ray of the chest. Yellow arrows show nodular opacities in the lungs.

facilitating prompt diagnosis and treatment. CT provides useful information regarding the size, number, location, and extent of pseudoaneurysms (2).

To our knowledge only one case of spontaneous regression of multiple pulmonary aneurysms has been reported in medical literature, but our case is the first one in patient with midaortic syndrome (3).

Address for correspondence: M. Kardos, MD, Children’s Cardiac Center, Limbova 1, SK-833 51 Bratislava, Slovakia.

Phone: +421.2.59371864, Fax: +421.2.54792317

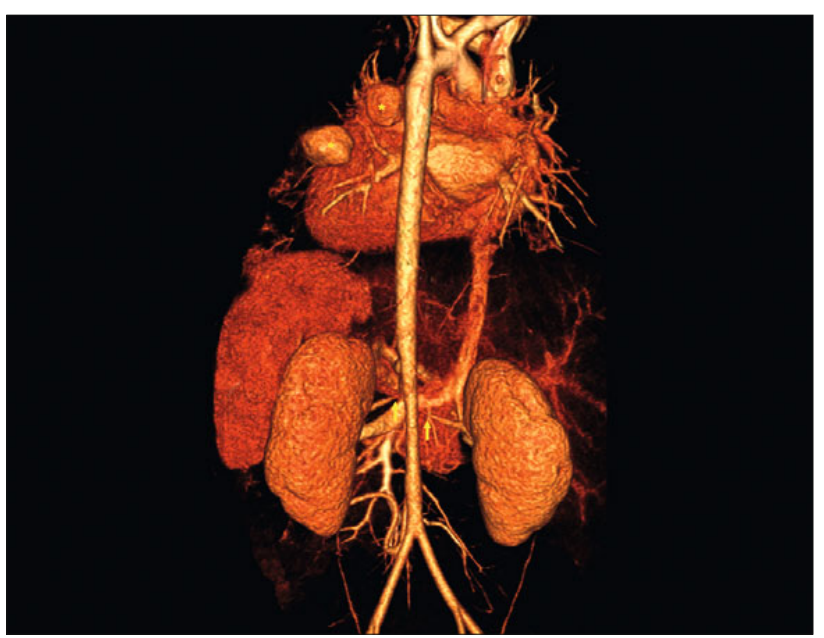

Fig. 2. VRT reconstruction of the whole aorta. Two big PAPs (yellow asterisk) are seen on the left side of the aorta, narrowing of the aorta in the abdominal course and severe stenosis of both renal arteries (yellow arrow).

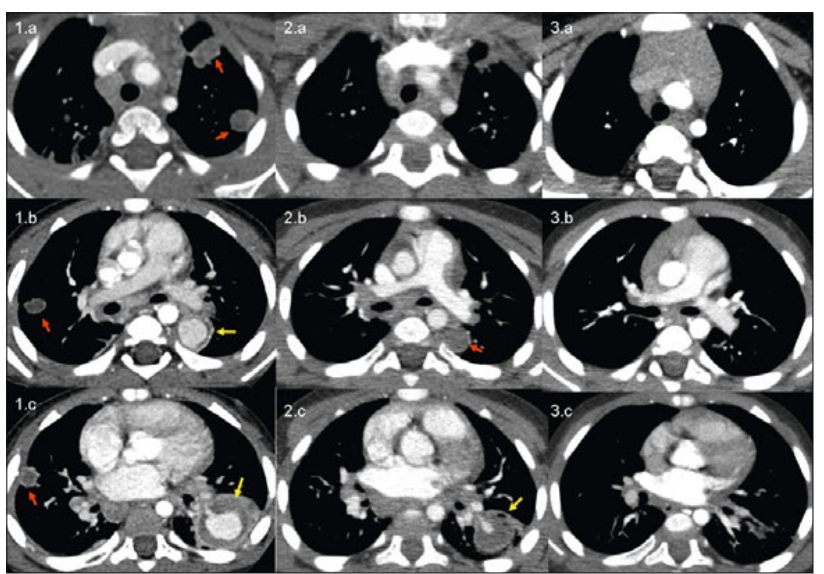

Fig. 3. CT angiography - same levels of CT scans in axial view. 1. First examination - multiple completely thrombosed (red arrows) and partially thrombosed (yellow arrows) pseudoaneurysms. 2. One month later-regression of pseudoaneurysm (PAPs), all of them thrombosed. 3. Seven months later - no PAPs are present. VRT reconstruction of the whole aorta. Two big PAPs (yellow asterisk) are seen on the left side of the aorta, narrowing of the aorta in the abdominal course and severe stenosis of both renal arteries (yellow arrow). 
119-120

A two-year-old boy was referred to our cardiology intensive care unit for severe pharmacoresistant arterial hypertension. Before admission, the patient overcame MRSA pneumonia. Due to the presence of multiple nodular opacities in lungs on chest X-ray, CT scans of both renal arteries and chest were carried out (Fig. 1). CT angiography confirmed the presence of severe stenosis of truncus coeliacus, superior mesenteric artery and both renal arteries with narrowing of abdominal aorta in length of $5 \mathrm{~cm}$ (Fig. 2). Multiple pulmonary arterial pseudoaneurysms were found in both lungs. Some of them were completely and some partially thrombosed. The medical staff decided not to embolise them but only to monitor the patient because no hemoptysis has occurred. The PAPs had completely regressed after 7 months (Fig. 3). Then the patient has undergone some balloon dilatations of renal arteries with partial success.

In our case we decided not to intervence these pseudoaneurysms for several reasons. On the first CT scan some of pulmonary arterial pseudoaneurysms were completely or partially thrombosed.
We supposed that the process of thrombotisation was going to continue. And no hemoptysis has occured. The patient had to be intensively monitored at the intensive care unit and radiologist had to be ready at any time manage this patient in case of acute severe hemorrhage.

\section{References}

1. Garnett GM, Kimball S, Kon K et al. Pulmonary artery pseudoaneurysm after MRSA septicemia in a pediatric patient. Journal of Pediatric Surgery. 2013; 48: 33-36.

2. Nguyen ET, Silva CI, Seely JM et al. Pulmonary Artery Aneurysms and Pseudoaneurysms in Adults: Findings at CT and Radiography. AJR. 2007; 188: 126-134.

3. Horwitz M, Chaumoitre K, Grimaldi C et al. Spontaneous Regression of Multiple Rasmussen Aneurysms in a Child With Lemierre Syndrome and Pulmonary Abscesses. Pediatric Infectious Disease Journal. 2013; 32:1301-1302. 